\title{
3D Printing: Towards the Future of Oral and Maxillofacial Surgery
}

\section{Marwa G Noureldin* and Noha Y Dessoky}

Lecturer, Department of Oral and Maxillofacial Surgery, Faculty of Dentistry, Alexandria University, Egypt

*Corresponding Author: Marwa G Noureldin, Lecturer, Department of Oral and Maxillofacial Surgery, Faculty of Dentistry, Alexandria University, Egypt.
Received: June 20, 2020

Published: June 30, 2020

(C) All rights are reserved by Marwa G

Noureldin and Noha Y Dessoky.

\section{Abstract}

This descriptive review focuses on the advanced revolution of the 3D printing technology in the field of oral and maxillofacial surgery (OMFS). This technology is now trending and growing popular throughout the different aspects of the specialty to include oral and maxillofacial diagnosis, treatment planning, surgical interventions and novel tissue engineering. Moreover, it has an interesting prospect in education and training of dental students.

Keywords: 3D Printing; 3D Planning; Oral and Maxillofacial Surgery; Tissue Engineering; Education

\section{Introduction}

The use of 3D printing also known as additive manufacturing or rapid prototyping in oral and maxillofacial surgery (OMFS) continues to show growing interest. 3D printing was initiated over thirty years ago. Today, this computerized revolution is gaining attention and popularity focusing especially on applications related to OMFS [1].

\section{Process for 3D printing-based tactile models}

Medical models or bio-models represent are obtained from 3D medical imaging such as computed tomography (CT) scan, cone beam computed tomography (СВCT) or magnetic resonance imaging (MRI). High quality volumetric 3D image data of the required anatomical structure can be obtained. Digital Imaging and Communications in Medicine (DICOM) format files are created and with the aid of computer aided design (CAD) software, converted to Standard Tessellation Language (STL) format files. These files are then uploaded into the 3D printer and a rapid prototyping (RP) model is then fabricated, guided by quality assurance of the model and ensuring its dimensional accuracy [2].

These previous steps require major expertise and understanding of medical imaging, 3D image processing and designing in addition to software manufacturing and engineering procedures. The construction of reliable, dimensionally accurate models necessitates a team of specialists as specialists in medical imaging, surgeons and engineers [3].

\section{Current 3D printing techniques}

Three-dimensional printing technology can be classified according to the techniques, aimed deposition process or the materials used. The techniques include fused deposition modeling (FDM), selective laser sintering (SLS), stereolithography (SL), Polyjet printing and bioprinting [4-6]. The aimed deposition process classification includes polyjet modeling based on drop-on-drop deposition, and fused deposition modeling based on continuous deposition [7].

The material classification includes thermoplastic, metal powder, ceramic powder, eutectic metals, alloy metal, photopolymer, paper, foil, plastic film, titanium alloys or even living cells in layers to produce a 3D object $[8,9]$. Only limited materials are compatible with the human body. 3D titanium-based implants were found to be the most acceptable causing no irritation to the recipient site [10].

Applications of 3D printing technology in OMFS

Preoperative treatment planning and simulation

Three-dimensional-virtual environment presents an important role in preoperative planning and surgery simulation. The virtual reality (VR) has the ability to rightfully contemplate the soft tissue and bony changes. It provides surgeons with the best possible scenario for preoperative treatment planning. It made it easy to plan and execute surgeries virtually with immediate loading of the prostheses [11]. These simulations allow surgeons to prepare the required instruments for execution of the procedure [12]. A simulated model of the planned outcome can be 3D printed, serving the 
patient to visualize the treatment outcome and also assisting in receiving the patient's consent before performing the surgery [13].

\section{Cranio-maxillofacial reconstruction}

Computer aided design-computer aided manufacturing (CADCAM) was first introduced to fabricate craniofacial anatomical models based on images from CT in 1987 [14]. A 3D printed model was used by Bill., et al. for planning of a surgery, in which an allogenic bone transplant was used for cranioplasty.

Skulls have irregular outlines, so it is tough to standardize a cranial implant [15]. Assessment of bony defects for grafting is crucial and throughout practice RP models were shown to be beneficial for recognizing the ideal donor site. For example, reconstruction of the skull can be accomplished using a split calvarial bone graft and the ideal donor area can be accurately located in advance to best harmonize with the ideal recipient calvarial bone curvature [16].

Three-dimensional titanium-based implants were found to be useful for the reconstruction of the calvarium and maxilla with excellent fit and design. The precise preoperative simulation serves to provide customized resection guides to accurately execute the resection $[10,17]$.

Three-dimensional printing technology now allows the fabrication of surgical guides to aid fibular osteotomy for its use as a graft with the help of fixation guides to enable maxillary reconstruction $[17,18]$.

Extensive resection of oral and maxillofacial tumors as mandibulectomy can be devastating for the patient. The reconstruction of the mandible to restore its contour can be quite challenging. Moreover, alteration in the alignment of the mandibular structure can negatively affect the function as a result of malocclusion [19].

Computer assisted simulation with the aid of CAD-CAM programs were able to solve these difficulties. A 3D printed RP model was developed and used to pre-bend titanium reconstruction plates. Excellent outcomes were reported from their use in mandibular reconstruction [20].

This technique also allowed accurate adaptation of the reconstruction plate therefore, undergoing minimal handling during the operation, preserving the strength of the plate and reducing operating time considerably. Moreover, excellent mandibular symmetry was easier to accomplish [21].

\section{Maxillofacial fractures}

Three-dimensional printing technology significantly impacted both the patients and the surgeons. Preoperative assessment of traumatic cases in addition to predesigning of customized fixation devices led to the production of precise 3D printed prothesis and implants for the treatment of traumatic defects. The surgery time, wound exposure time are reduced and postoperative complications were lowered [22].

Dessoky N., et al. have used 3D planning for virtual reduction of mandibular fractures and milling for the production of custommade poly-ether-ether ketone (PEEK) plates. They showed precise and accurate results which led to reduced operating time since the plates were previously pre-bent showing successful surgical outcomes [23].

Furthermore, the custom-made prosthesis has shown high accuracy thus improving the patient's esthetics as well as the psychological status since it resulted in correct symmetry of the facial contour [13]. The mirror imaging of the unaffected contralateral side can impressively improve the accuracy of the reconstruction [24].

A 51 years old patient had a delayed craniofacial reconstructive surgery after trauma at Lake Hospital in Baton Rouge, but it resulted in a residual defect in the fronto-orbital region and an inferiorly displaced zygomatico-maxillary complex with increased orbital volume. The defect couldn't be reconstructed with an ocular prosthesis, leaving the patient with an unesthetic defect. A 3D CT was done and a stererolithographic model was printed for better assessment of the defect and a custom-made PEEK implant was fabricated and put in place. The patient was left with a reliable, functional and esthetic result [25].

Surgical guides and templates for guided implant placement

Due to the advances in 3D CT and the computer-based software programs, surgeons have developed their strategies in the treatment planning phase. Remarkably, it also allows surgeons to do the surgery virtually and to check the treatment options. Also, saving time for planning the surgery and improving precision [26].

Rapid prototyping means producing models of anatomical areas of interest accurately from CAD data $[27,28]$. CAM software are used to make implant surgical guides and anatomic models. Images obtained from CT are transferred to the computer to plan the surgical guide with a computer software. 3D models made of acrylic resin and surgical guides are then printed. Once it is set, the guides have spaces for stainless steel drill-guiding tubes. Metal cylinders are put into the spaces, and then the guides are ready to be used clinically [29].

The used sleeves in the surgical guide are for accurate drilling and implant placement. This gives good results for the patient and 
the clinician. The existing bone can be used wisely and bone grafting avoided. The implant position can be placed in the best angulations, position and depth by merging the digital data of the bone with the planned restoration. A provisional or permanent restoration can be fabricated preoperatively on the cast which can be used as an immediate provisionalization. The outcomes of this procedure are predictable and precise, and hence the final restoration [30].

Fabrication of custom prosthesis for orthognathic surgery and dentofacial deformities

Personalized orthognathic surgical guides (POSG) can be manufactured using 3D printing technique preoperatively which allows accurate osteotomies, positioning of bone segments, drill holes and screws. The custom titanium plates can be placed only if the bony segments are in the correct position, ensuring the right placement of bone segments [31].

Three-dimensional printed models have been popularly used for planning distraction osteogenesis (osteotomies, vector of distraction). The ability to customize fixation plates of a distractor based on the 3D printed model. The plates can be easily adapted during surgery for their high accuracy of fit at the predicted positions. Also, parallel alignment the connecting pins was facilitated, thus confirming the proper transmission of distraction forces [32].

Three-dimensional printing technology can also help in correcting craniosynostosis by providing osteotomy guides that are very helpful in the process of reconstruction. Additionally, the surgery can be simulated by 3D tactile models [33]. Le Forte I, II, or III midface osteotomies can be simulated effectively using 3D printed prototypes models, as they need delicate blind osteotomy [34].

Three-dimensional printed models permit planning and testing of orthognathic surgeries preoperatively. Evaluation of the relationship between proximal and distal mandibular segments after bilateral sagittal split osteotomies became applicable. These planned movements in addition to the fabrication of osteotomy guides as well as occlusal splints increased the accuracy during operation [35].

As for genioplasties, the ability to create a surgical guide for transferring osteotomies from the 3D printed model to the operation in addition to pre-bending of the titanium plates on 3D models can deliver accurate results intraoperatively [3].

Computer-assisted genioplasty by combining the use of 3D cephalometry, a 3D RP model, and pre-bent titanium plates was described to have a valuable outcome [7]. However, 3D printed mod- els can show some errors related to accuracy, which can affect the ideal dental occlusion in orthognathic surgery. Consequently, scanning devices are indicated to obtain ideal dental occlusal splints particularly for regions involving dentition [36].

\section{Customized temporomandibular joint (TMJ) reconstruction}

Some TMJ pathologies involve the condyle and the ramus of the mandible. These conditions require joint resection followed by total joint prosthesis with reliable treatment outcomes [37].

At Melbourne University, a 3D printed prosthesis was fabricated for a 32-year-old patient who lost a part of his left mandible involving the condyle, following a childhood trauma. The functional status of the patient was compromised causing him pain and affecting his chewing ability. The 3D-printed prosthesis was composed of a titanium part and a plastic jaw joint. For the condylar part, the titanium resembled the patient anatomical condyle. Using the CT scan images, the technicians also printed a 3D plastic model of the patient's skull and then the titanium part was refined to attain a flawless fit. With the aid of computer simulations, the printed prosthesis was subjected to loading forces mimicking the actual loads to test the prosthesis and to guarantee that it doesn't fail during function [38].

In a study by $\mathrm{C}$ Lee Ventola who created a custom 3D titanium TMJ for a patient with a history of sclerodema. The patient suffered spontaneous bilateral pathologic fracture of the mandible which resulted in an open bite. The 3D custom made prostheses was successful alleviating the patient's agony [39].

\section{Fabrication of tissue engineered scaffolds}

Three-dimensional printing has been used in experimental approaches such as bone grafting in reconstructive surgery. With the known types of available grafts; autogenous, autologous and allogenic grafts, the allogenic grafts are the best used since they have no ethical, infection and size limit and no donor site morbidity problems. On the other hand, they don't have the gold standards of the autogenous grafts which have osteogenic and osteoinductive properties [40].

Thanks to the novel technology, 3D printing technique has adequately evolved bio-cell printing for creation of 3D scaffolds for tissue engineering [6]. The triad for tissue engineering process encompasses cell, scaffold, and growth factor. Using this technology, reconstruction of the fracture regions or other mandibular deficient areas can be accomplished. These scaffolds were used to generate bone and tissue using biocompatible material. The scaffolds can be accurately fabricated according to the required shape and 
dimensions predesigned by the 3D planning. In a study by Mehra., et al. a portion of autogenous bone was obtained from a patient suffering from osteoporosis and subsequent mandibular fractures. These cells were then isolated, and cultivated in a special laboratory, then incorporated in a customized biodegradable poly (D, Llactic-coglycolic acid) (PLGA) scaffold which was constructed according to the existing defect at the time of reconstructive surgery. Afterwards, this 3D bio-cell printed scaffold was secured to the patient's mandible with rigid fixation. The postoperative radiographs showed healing of the fractures, a well incorporated scaffold with new bone formation and an overall increase in bone volume [41].

Also, the properties of the scaffold such as porosity, texture and design are all easily adjustable by the program. Another advantage was the ability to add osteoinductive factors like bone morphogenic proteins to stimulate osteogenic differentiation thus increasing the integration between the printed scaffold and the recipient bone [42].

Three-dimensional printed cell culture for tissue engineering were developed to produce artificial tissues in vitro models $[43,44]$ Incorporating stem cells into the 3D cultures for transplantation and tissue regeneration was achievable [45]. 3D printed cell cultures aided in the formation of sufficient human bone as well as skin grafts in vitro [46].

Providing an educational tool for medical students and residents.

Generating a three-dimensional model by 3D printing were easily manufactured to be used for education and have been helpful to dental students [47]. Three-dimensional printing can replicate the soft and hard tissue with high precision resulting in a training jaw for students to design incisions and practice surgical resections $[48,49]$.

Fabrication of customized medical protective equipment

Even though 2020 has been a time of crisis due to the spread of Covid 19 virus. Many individuals spent the lockdown time searching for ways to benefit medical staff and dentists around the world. With 3D printers being accessible in several homes and centers, time was spent fruitfully bringing 3D printed face masks $\mathrm{N} 95$ respirators and face shields to light. The masks were fabricated from a more durable material with longer shelf lives than the ordinary ones which require constant changing of the filters. Moreover, fabrication of various sizes of the frame was feasible. To our knowledge, this is the first time for $3 \mathrm{D}$ medical protective equipment to be reported [50].

\section{Conclusion}

In conclusion, 3D printing technology has revolutionized the field of OMFS in many aspects. It saves time and effort with eminent outcomes. Also, it's growing popular for its ability to produce personalized implants and devices with high accuracy and fit. This novel and trendy technique is now favored by many dentists and is increasingly appealing to various patients.

\section{Bibliography}

1. Oberoi Gunpreet., et al. "3D Printing-Encompassing the Facets of Dentistry". Frontiers in Bioengineering and Biotechnology 6 (2018).

2. Marro Alessandro., et al. "Three-Dimensional Printing and Medical Imaging: A Review of the Methods and Applications". Current Problems in Diagnostic Radiology 45.1 (2016): 2-9.

3. Olszewski R., et al. "Innovative Procedure for Computer-Assisted Genioplasty: Three-Dimensional Cephalometry, RapidPrototyping Model and Surgical Splint". International Journal of Oral and Maxillofacial Surgery 39.7 (2010): 721-724.

4. Visscher Dafydd O., et al. "Advances in Bioprinting Technologies for Craniofacial Reconstruction". Trends in Biotechnology 34.9 (2016): 700-710.

5. Moroni Lorenzo., et al. "Biofabrication: A Guide to Technology and Terminology". Trends in Biotechnology 36.4 (2018): 384402.

6. Zhang Yu Shrike., et al. "3D Bioprinting for Tissue and Organ Fabrication". Annals of Biomedical Engineering 45.1 (2017): 148-163.

7. Raphael Olszewski and Reychler Herve. "Clinical Applications of Rapid Prototyping Models in Cranio-Maxillofacial Surgery". Advanced Applications of Rapid Prototyping Technology in Modern Engineering, IntechOpen (2011).

8. Schubert Carl., et al. "Innovations in 3D Printing: A 3D Overview from Optics to Organs". The British Journal of Ophthalmology 98.2 (2014): 159-161.

9. "Science and Society. Experts Warn against Bans on 3D Printing”. Science (New York, N.Y.) 342.6157 (2013): 439.

10. Chen Shuo-Tsung., et al. "3-D Titanium Mesh Reconstruction of Defective Skull after Frontal Craniectomy in Traumatic Brain Injury". Injury 46.1 (2015): 80-85. 
11. Wang Yu., et al. "Reconstruction of Mandibular Contour Using Individualized High-Density Porous Polyethylene (Medpor®) Implants Under the Guidance of Virtual Surgical Planning and 3D-Printed Surgical Templates". Aesthetic Plastic Surgery 42.1 (2018): 118-125.

12. Jacobs Carly A and Alexander Y Lin. "A New Classification of Three-Dimensional Printing Technologies: Systematic Review of Three-Dimensional Printing for Patient-Specific Craniomaxillofacial Surgery". Plastic and Reconstructive Surgery 139.5 (2017): 1211-1220.

13. Wilde F and A Schramm. "[Computer-aided reconstruction of the facial skeleton: Planning and implementation in clinical routine]". HNO 64.9 (2016): 641-649.

14. Brix F and J T Lambrecht. "[Preparation of individual skull models based on computed tomographic information]". Fortschritte Der Kiefer- Und Gesichts-Chirurgie 32 (1987): 74-77.

15. "Skull Joints". (2020). Kenhub. www.kenhub.com. https:// www.kenhub.com/en/library/anatomy/the-skull-joints.

16. Lee Hee Jong., et al. "Secondary Skull Reconstruction with Autogenous Split Calvarial Bone Grafts versus Nonautogenous Materials". The Journal of Craniofacial Surgery 25.4 (2014): 1337-40.

17. Biron Vincent L., et al. "Radial Forearm Free Flap with Titanium Mesh Sandwich Reconstruction in Complex Anterior Skull Base Defects". The Journal of Craniofacial Surgery 23.6 (2012): 1763-65.

18. Lethaus Bernd., et al. "Reconstruction of a Maxillary Defect with a Fibula Graft and Titanium Mesh Using CAD/CAM Techniques". Head and Face Medicine 6 (2010): 16.

19. Abou-ElFetouh Adel., et al. "Computer-Guided Rapid-Prototyped Templates for Segmental Mandibular Osteotomies: A Preliminary Report". The International Journal of Medical Robotics + Computer Assisted Surgery: MRCAS 7.2 (2011): 187192.

20. Salgueiro Martin I and Mark R Stevens. "Experience with the Use of Prebent Plates for the Reconstruction of Mandibular Defects". Craniomaxillofacial Trauma and Reconstruction 3.4 (2010): 201-208.
21. Thankappan Krishnakumar., et al. "Three-Dimensional Computed Tomography-Based Contouring of a Free Fibula Bone Graft for Mandibular Reconstruction". Journal of Oral and Maxillofacial Surgery: Official Journal of the American Association of Oral and Maxillofacial Surgeons 66.10 (2008): 2185-2192.

22. Ursan Iulia D., et al. "Three-Dimensional Drug Printing: A Structured Review". Journal of the American Pharmacists Association: JAPHA 53.2 (2013): 136-144.

23. Dessoky Noha Y., et al. "USE OF CUSTOM MADE PEEK PLATES FOR TREATMENT OF MANDIBULAR FRACTURE”. Alexandria Dental Journal, Alexandria University; Faculty of Dentistry (2020).

24. Aleid Wesam., et al. "Development of In-House Rapid Manufacturing of Three-Dimensional Models in Maxillofacial Surgery". The British Journal of Oral and Maxillofacial Surgery 48.6 (2010): 479-481.

25. Patel Neel., et al. "Use of Virtual Surgical Planning for Simultaneous Maxillofacial Osteotomies and Custom Polyetheretherketone Implant in Secondary Orbito-Frontal Reconstruction: Importance of Restoring Orbital Volume". The Journal of Craniofacial Surgery 28.2 (2017): 387-390.

26. Benavides,Erika., et al. "Use of Cone Beam Computed Tomography in Implant Dentistry: The International Congress of Oral Implantologists Consensus Report”. Implant Dentistry 21.2 (2012): 78-86.

27. Drstvensek Igor., et al. "Applications of Rapid Prototyping in CranioMaxilofacial Surgery Procedures". International Journal of Biology and Biomedical Engineering 2.1 (2008).

28. Faber Jorge., et al. "Rapid Prototyping as a Tool for Diagnosis and Treatment Planning for Maxillary Canine Impaction". American Journal of Orthodontics and Dentofacial Orthopedics: Official Publication of the American Association of Orthodontists, Its Constituent Societies, and the American Board of Orthodontics 129.4 (2006): 583-589.

29. Sarment D and C Misch. "Scannographic Templates for Novel Pre-Implant Planning Methods". (2002): 16-22.

30. Job Shibu., et al. "In Vivo Evaluation of Crestal Bone Heights Following Implant Placement with 'flapless' and 'with-Flap' Techniques in Sites of Immediately Loaded Implants". Indian Journal of Dental Research: Official Publication of Indian Society for Dental Research 19.4 (2008): 320-325. 
31. Li B., et al. "A New Approach of Splint-Less Orthognathic Surgery Using a Personalized Orthognathic Surgical Guide System: A Preliminary Study". International Journal of Oral and Maxillofacial Surgery 46.10 (2017): 1298-1305.

32. Klammert Uwe., et al. "Multi-Directional Le Fort III Midfacial Distraction Using an Individual Prefabricated Device". Journal of Cranio-Maxillo-Facial Surgery: Official Publication of the European Association for Cranio-Maxillo-Facial Surgery 37.4 (2009): 210-215.

33. Choi Jong W., et al. "One-Piece Frontoorbital Advancement with Distraction but without a Supraorbital Bar for Coronal Craniosynostosis". Journal of Plastic, Reconstructive and Aesthetic Surgery: JPRAS 62.9 (2009): 1166-1173.

34. Choi Jong Woo., et al. "Use of Distraction Osteogenesis to Change Endocranial Morphology in Unilateral Coronal Craniosynostosis Patients". Plastic and Reconstructive Surgery 126.3 (2010): 995-1004.

35. Mavili Mehmet Emin., et al. "Use of Three-Dimensional Medical Modeling Methods for Precise Planning of Orthognathic Surgery". The Journal of Craniofacial Surgery 18.4 (2007): 740-747.

36. Zinser Max J., et al. "Computer-Assisted Orthognathic Surgery: Feasibility Study Using Multiple CAD/CAM Surgical Splints”. Oral Surgery, Oral Medicine, Oral Pathology and Oral Radiology 113.5 (2012): 673-687.

37. Wolford Larry M and Pushkar Mehra. "Custom-Made Total Joint Prostheses for Temporomandibular Joint Reconstruction". Proceedings (Baylor University. Medical Center) 13.2 (2000): 135-138.

38. Australian-First Reconstructive Surgery Uses 3D Printed Jaw Implant Manufactured at the University of Melbourne|Study Options.

39. Ventola C Lee. "Medical Applications for 3D Printing: Current and Projected Uses". P and T: A Peer-Reviewed Journal for Formulary Management 39.10 (2014): 704-711.

40. Hikita Atsuhiko., et al. "Bone Regenerative Medicine in Oral and Maxillofacial Region Using a Three-Dimensional Printer<sup/>”. Tissue Engineering. Part A 23.11-12 (2017): 515-521.
41. Mehra Pushkar., et al. "Use of 3-d Stereolithographic Models in Oral and Maxillofacial Surgery". Journal of Maxillofacial and Oral Surgery 10.1 (2011): 6-13.

42. Tsai Kuo-Yang., et al. "Laser Sintered Magnesium-Calcium Silicate/Poly- $\varepsilon$-Caprolactone Scaffold for Bone Tissue Engineering". Materials 10.1 (2017).

43. Knowlton Stephanie., et al. "Bioprinting for Cancer Research". Trends in Biotechnology 33.9 (2015): 504-513.

44. Athirasala Avathamsa., et al. "A Dentin-Derived Hydrogel Bioink for 3D Bioprinting of Cell-Laden Scaffolds in Regenerative Dentistry". Biofabrication 10.2 (2018): 024101.

45. Murphy Sean V and Anthony Atala. "3D Bioprinting of Tissues and Organs". Nature Biotechnology 32.8 (2014): 773-785.

46. Almela Thafar., et al. "3D Printed Tissue Engineered Model for Bone Invasion of Oral Cancer". Tissue and Cell 52 (2018): 7177.

47. Marconi Stefania., et al. "Value of 3D Printing for the Comprehension of Surgical Anatomy". Surgical Endoscopy 31.10 (2017): 4102-4110.

48. Lambrecht J Th., et al. "Generation of Three-Dimensional Prototype Models Based on Cone Beam Computed Tomography". International Journal of Computer Assisted Radiology and Surgery 4.2 (2009): 175-180.

49. Yusa Kazuyuki., et al. "Three-Dimensional Printing Model as a Tool to Assist in Surgery for Large Mandibular Tumour: A Case Report”. Journal of Oral and Maxillofacial Research 8.2 (2017).

50. Download Free 3D Printing Models Reusable Facial Mask Respirator Frame Cover Cults.

\section{Assets from publication with us}

- Prompt Acknowledgement after receiving the article

- Thorough Double blinded peer review

- Rapid Publication

- Issue of Publication Certificate

- High visibility of your Published work

Website: www.actascientific.com/

Submit Article: www.actascientific.com/submission.php Email us: editor@actascientific.com

Contact us: +919182824667 